\title{
ON THE VARIATIONS
}

IN THE AMOUNT OF

\section{SUIPHOCYANIDE OF POTASSIUM}

IN THE

\section{SALIVA OF PERSONS AFFECTED WITH DIFFERENT DISEASES.}

BY

SAMUEL FENWICK, M.D., F.R.C.P., PHYsician to THE LONDON HOSPITAL.

(Received January 6th-Read March 28th, 1882.)

IT being known that both the colouring matter and the salts of the bile can be occasionally detected in the saliva in cases of disease, the thought was naturally suggested that perhaps, under normal conditions, the saliva might contain some substance resulting from the decomposition which the biliary acids are believed to undergo. It is well known that the saliva contains sulphocyanide of potassium, which is easily reognised by the red colour produced on the addition of a persalt of iron; and as the sulphocyanide is met with only in the saliva, as it contains sulphur in an unoxidised form, and as it is known to vary greatly in amount in different cases, it was selected for observation.

The amount of the sulphocyanide is so small that a quantitative test is a matter of great difficulty; it was 
therefore thought necessary to trust to the depth of colour produced by the addition of a weak solution of perchloride of iron. The saliva of each person examined was collected as it flowed from the mouth for about two minutes; and seven minims of a solution of half a drachm of the iron to an ounce of water were added, and the colour thus obtained was compared with a scale of colours prepared in the following manner :

A quantity of saliva was collected from a number of healthy persons, and the tint produced by the addition of the perchloride of iron was taken as the normal amount; a portion of the same saliva was then evaporated to a quarter and another to half its bulk, and the colours produced in these respectively were assumed to represent four times and twice the normal quantity of sulphocyanide; while two other portions diluted with water to twice and four times their amount were taken as representing half and a quarter of the normal quantity of the salt.

In this way I examined, during the course of many years, the saliva of nnmerous persons who consulted me for different maladies, recording also the symptoms of which they complained, and in most cases the results of the treatment adopted.

These observations were so numerous (probably many thousands) that I selected for analysis only those in which the amount of the sulphocyanide was much below or much above the normal standard. In the following pages these are described as "private cases."

In order to check these observations I requested Dr. Needham, at that time house physician to the London Hospital, to examine the saliva of a number of patients who were under treatment in the wards, and two years afterwards a similar inquiry was carried on by Dr. Bedford Fenwick in the same hospital.

The cases thus obtained, numbering between 200 and 300 , have been analysed together, and are described as "hospital cases."

As it has been asserted by some physiologists that the 
presence of the sulphocyanide of potassium in the saliva is accidental, and is the result of decomposition set up by decayed teeth, it seemed desirable, in the first place, to ascertain how far such an opinion is supported by facts.

Careful inquiries were made as to the state of the teeth in 87 cases in the hospital, and with the following results. The teeth were quite perfect in 18 per cent. of those whose saliva contained a normal or excessive amount of sulphocyanide, and in only 14 per cent. of those in whom it was deficient. One or two teeth were carious in 47 per cent. of the former and in 44 per cent. of the latter; whilst many were decayed in 42 per cent. of those in whose saliva the sulphocyanide was below, and in 35 per cent. of those in whom it was normal or excessive. It is evident, therefore, as the salt presents itself in excessive quantities where there is no decay of the teeth, and is as often deficient as superabundant where many teeth are carious, the opinion before mentioned must be incorrect.

Others have attributed the presence of sulphocyanide to the smoking of tobacco, and the habits of 213 patients in the hospital were investigated as to this point. As the greater number had been confined to their beds in wards in which smoking was not allowed, only a few of them had latterly enjoyed the opportunity of using tobacco. On analysing these cases it appeared that of those whose saliva contained an excess of sulphocyanide 6 per cent. only had latterly smoked; of those presenting a normal quantity, only 2 per cent.; whilst of those in whom there was a deficiency, 8 per cent. had latterly consumed tobacco, and one represented himself as an excessive smoker. It is evident, therefore, that no material influence is exerted by the use of tobacco, although my own impression, before analysing these cases, was that the sulphocyanide is usually present in greater quantity in the saliva of those who smoke than of those who abstain from tobacco.

I propose, in the first place, to examine only those 
cases in which the sulphocyanide was deficient, and to see what conclusions can be drawn from them.

One of the most striking points that presents itself in these cases is the frequency with which it is below the normal amount in jaundice arising from obstruction. Thus, of 23 persons admitted into the hospital with this disorder, it was deficient in 18, and in many of them not even a trace of colour could be obtained by the addition of the perchloride of iron. It 2 the saliva was normal, but one of these had so far recovered as to have been placed on full diet, and in the other postmortem examination proved the jaundice to have resulted from the rupture of a hydatid cyst into the ducts of the liver, the opening of the common duct being only partially obstructed, and bile being present in the duodenum. In 1 case out of 3 in which the sulphocyanide was in excess the jaundice was probably due to partial obstruction of the ducts of the liver by carcinomatous growths; the histories of the others could not be obtained.

From these facts it would seem probable that in order that the sulphocyanide should appear in the saliva, the bile must be able to enter the duodenum, and this seems to be supported by 2 cases admitted into the London Hospital, in which large quantities of bile were discharged through the lungs, probably from rupture of hydatid cysts of the liver; and in both of which scarcely any colour was developed in the saliva by the addition of perchloride of iron.

Pointing to the same conclusion is the circumstance that in some cases of jaundice the depth of the colour of the stools varies with the amount of the sulphocyanide. Thus, a lady had suffered three weeks from jaundice, arising apparently from hypertrophic cirrhosis; the skin and urine were deeply tinged, the stools white, and the saliva free from sulphocyanide. She was treated with perchloride of mercury, and in three weeks the stools became partially coloured and a slight tinge was produced in her saliva by the addition of perchloride of iron. 
In two weeks afterwards diarrhœa occurred, the stools being deeply stained with bile, and although the skin and urine remained yellow, the saliva contained an abundance of the sulphocyanide.

CASE 2.-A gentleman had been for three months deeply jaundiced, the saliva being -2. In two weeks afterwards the jaundice had greatly lessened, the stools had become brown coloured, and the saliva was +2 . Two or three weeks after this he had a severe attack of pain in the abdomen, with an increase of the jaundice, and the sulphocyanide again became deficient. When I last saw him he had been much better, and the sulphocyanide was again +2 .

In severe cases of jaundice arising from obstruction, and it must be remembered that only severe cases are admitted into the hospital, the deficiency of the sulphocyanide has been usually greatest at the beginning of the disease. Thus, in all excepting one, which was of three months' standing, where there was a complete absence of the sulphocyanide $(-8)$, the saliva was examined within one week of the coloration of the skin, whilst in those whose average duration had been five weeks the saliva was -4 .

This rule, however, does not hold good for those temporary cases of jaundice following severe abdominal pain, and usually supposed to arise from gall-stones. In two such instances the saliva was normal, and in each the jaundice passed away in a day or two. If these observations should be confirmed by subsequent inquiries we shall have in the saliva a valuable means of prognosis; for when the sulphocyanide is completely absent at the outset of the disease, the case will be probably more obstinate than where some can be discovered. In chronic cases (for example, after three months' duration) I have generally found that when the sulphocyanide was permanently absent the prognosis was most unfavorable, but when its presence could be still discovered the obstruction was incomplete and recovery often took place. 
Where there is no obstruction to the entrance of the bile into the duodenum, one of the chief circumstances which seems to determine the amount of the sulphocyanide is the quantity of food, and especially of animal food, that is taken into the stomach and digested. For example, in all the cases of obstruction of the cesophagus I have examined, there has been from the first a marked lessening in the amount of colour produced by the addition of the perchloride of iron, and this has progressively increased as the difficulty of taking food has augmented.

In eight cases of cancer of the stomach the sulphocyanide has been very scanty or entirely absent.

In connection with this it is worthy of remark that physiologists have come to the conclusion, from experiments on animals, that the amount of the bile secreted is mainly regulated by the quantity of food that is taken, and that when food is altogether withheld the secretion of bile rapidly decreases.

Of course persistent vomiting must lessen the amount of food digested as certainly as if only a small quantity were given, and we find that in 111 of the "private cases," where there was a marked deficiency of the sulphocyanide, 29 were suffering from a more or less constant rejection of the contents of the stomach.

Long-standing diarrhœa and dysentery seem also to act like vomiting, in lessening the amount of the sulphocyanide; for in 23 out of 111 "private cases," where it was deficient, one of these conditions was present, but a temporary attack of diarrhœa seldom produced much alteration. It is interesting to observe that Dr. Rutherford, when reviewing the results of his experiments on the secretion of bile in dogs, asserts that "we have, however, found several drugs that have an indirectly depressant action; thus, when the intestinal glands are excited to secrete, there is an indirectly depressant effect on the liver, whereby the bile secretion is lessened. We invariably observed that while slight purgation by a purely intestinal irritant scarcely, if at all, depressed the secretion of 
the bile, powerful purgation produced a very marked effect."l

As the sulphocyanide has been shown to be deficient whenever the supply of food has been small, it might be surmised that this condition would also present itself in atonic dyspepsia, in which disease the digestion of the food is so imperfect. This is in fact by far the most frequent cause, for 39 out of 111 "private cases" were instances of this disorder. They were all very severe cases, the appetite being bad in almost all, and in many the sensation of hunger being entirely absent, or replaced by loathing of all food.

It is probable that feebleness of the digestive powers is also the most common cause of the deficiency of the sulphocyanide in various chronic maladies; for in 84 persons presenting this condition, in whom this point was carefully investigated, the appetite was stated to be bad in 55 cases, moderate in 12, and good in only 17 of the whole number.

The general nutrition of the body was also very defective; for of 53 cases, where the sulphocyanide was below the normal amount, 40 are stated to be thin, 3 moderately stout, and only 10 stout. In some of the latter the deficiency in the sulphocyanide was only temporary, and of the remainder, 4 are stated to be losing flesh.

There is, however, one class of cases which cannot be referred either to a want of food or to a deficiency of the digestive power. In every severe case of lead poisoning the sulphocyanide was absent or present in very small quantity, the deficiency persisted so long as the symptoms were urgent, and it was succeeded by an excess as soon as convalescence began.

This was so invariably the case that $I$ have been in the habit of employing it as a test of the condition of the patient, and have found it valuable in diagnosis and prognosis.

1 Prof. Rutherford, 'On the Action of Drugs on the Secretion of Bile,' p. 170. 
The cause of this circurnstance seems capable of ex. planation in two different ways. At first I supposed that, as the saliva was always very thick and tenacious, the lead acted as an astringent to the salivary glands, but I found that the deficiency of the sulphocyanide remained, even when these were stimulated to increased secretion by the application of tincture of pyrethrum to the tongue. A more probable hypothesis seems to be that the lead acts as a sedative upon the liver and prevents or lessens its secretion.

This view is confirmed by the experiments of Dr. Rutherford, who states that, "It cannot fail to strike the reader as a remarkable fact that while in the long list of drugs whose hepatic effect we have investigated we have found so many that stimulate the liver, there is only one'acetate of lead'-which appears to have a directly depressant effect."

It might naturally be expected that the secretion of sulphocyanide would be often modified by affections of the salivary glands themselves, but these glands are so little liable to disease that this seems seldom to be the case. I have, however, seen some cases of acute stomatitis in which no colour could be produced in the saliva by the perchloride of iron, but it is more probable that this arose rather from the inability of the patients to take food than from the effect of the inflammation of the mouth upon the glandular secretion.

In reviewing the circumstances that have appeared chiefly to coincide with a deficiency of the sulphocyanide in the saliva, we have found-

1. Any obstruction to the free entrance of the bile into the duodenum.

2. Any circumstance that diminishes the amount of food that is digested or absorbed, such as $(a)$ œsophageal stricture, (b) gastric cancer, (c) atonic dyspepsia, (d) persistent vomiting, $(e)$ long-standing diarrhca.

3. The effects of lead poisoning.

The two latter circumstances are, as has been shown, 
recognised by physiologists as the chief agents that in health tend to diminish the secretion of bile.

I propose now to inquire into the circumstances under which the sulphocyanide presents itself in the saliva above the normal quantity.

If the previous conclusions be correct, we should expect that an excess of the sulphocyanide would be found in conditions the reverse of those in which it was deficient: and such appears to be the case. Thus, in contrust to the figures before quoted as to the loss of appetite when the amount was below the normal, we find in 67 per cent. of the "private cases" whose saliva presented an excess that the appetite was good, in only 33 per cent. was it at all defective, and in no case was an absence of appetite a subject of complaint. Again, in opposition to the fact that most of those who had a deficiency of the sulphocyanide were thin or losing flesh, only 6 per cent. of those presenting an excess were thin, 20 per cent. were moderately stout, and 74 per cent. are recorded as "fat."

It was shown before that there is no good reason to believe that the amount of the sulphocyanide is in proportion to the quantity of bile secreted, and that this again depended in a large measure upon the amount of animal food digested and absorbed; so we may now conclude that an excess of the sulphocyanide represents an excess of nutritive material beyond what is necessary for the requirements of the system. The practical value of such an inference will be readily appreciated, and I have been in the habit of regulating the diet of dyspeptics in accordance with it. Where the sulphocyanide has been below the normal a liberal amount of animal food with alcoholic stimulants has been prescribed, but when it has been in excess a reduction in the quantity of food and stimulants, or an entire absence of alcohol, has been found to be beneficial.

We are not, however, by reference to the amount of food digested, able to explain all the cases in which the sulphocyanide is in excess, for it has presented itself in 
various diseases in which the patients were restricted to a very meagre diet.

This was especially observable in acute rheumatism. Of 36 cases of this disease admitted into the hospital all but one, whose saliva was normal, showed a marked excess of sulphocyanide, the amount varying from eight times to twice the ordinary quantity. The depth of colour also varied at different periods of the malady; those examined within seren days of the commencement of the fever presenting an average of $+4 \cdot 8$, in the second week it reached +6 , in the third week it fell to +4 , and in the fourth to $+3 \frac{1}{2}$.

The excess corresponded, in some degree, with the temperature and the pulse ; those having a saliva +2 presented an average pulse of 81 and an average temperature of $100.6^{\circ}$; those +4 a pulse of 92 and a temperature of $100.9^{\circ}$; whilst such as had +8 are recorded as having an average temperature of $101.3^{\circ}$ and a pulse of 101 .

This persistence of an excess of the sulphocyanide and its gradual decrease in acute rheumatism is interesting in regard to the effects of the salicylic acid upon the disease. Although the fever and the pains of the joints rapidly subside under its use, relapses are so apt to occur that the average duration of the patients in the hospital is not materially lessened.

It would seem probable from the persistence of an excess of the sulphocyanide that the fever runs a definite course, as is the case with the infectious febrile diseases. The saliva is always very acid in acute rheumatism, and I have found the patient is liable to relapses so long as his saliva is in an abnormal condition.

It is probable that persons who have habitually a large amount of sulphocyanide in their saliva are more liable to local rheumatism than others; for amongst the "private cases" 40 per cent. of those who had an excess of it had suffered from pains of the limbs or joints; whilst neuralgic affections were more common amongst those whose saliva presented a deficiency. 
All the cases of gout in the "hospital cases" had an excess of sulphocyanide, although not to so great an extent as in rheumatic fever; in the "private cases" there were 17 in whom it was above the normal, and there were none amongst those who exhibited a deficiency. The amount seems often to increase just before an attack, and I have on several occasions been able to predict a fit of gout by observing this.

In that large class of cases so commonly regarded as "gouty," but where there had been no articular affection, the saliva generally presented an excess of the sulphocyanide, and thus furnished a valuable indication for treatment. In almost every case of eczema and urticaria the same condition of the saliva was discovered, and it was also usually present in the various affections to which gouty persons are so liable.

Headache was one of the most common complaints to which those having an excess were subject, and the socalled "bilious headaches" constituted 25 per cent. of the whole number of "private cases," where this condition of the saliva was present. Persons suffering in this way usually belonged to families in which other members had suffered from gout or rheumatism; the attacks were periodical, and were often followed by a temporary improvement in health.

One tenth of those producing a deficiency also complained of " bilious headaches," but there was usually no gouty history, no relief was afforded by the attack, and the pain seemed to be of a more purely neuralgic character. The importance of such a distinction in a practical point of view need not be pointed out.

But if the sulphocyanide is so constantly in excess in acute rheumatism and gout, are we justified in regarding these diseases as produced by an excess of the sulphocyanide or the presence of some other sulphur compound in the system?

It is true that where it is in excess there is also very often an excess of lithic acid in the urine, but the excess 
of sulphocyanide occurs in so many different disorders of a total different character that such a hypothesis cannot be supported.

In the early stage of almost all febrile and inflammatory affections the sulphocyanide is usually in excess. For example, in gastric catarrh this condition is almost always present; thus, 27 per cent. of the private cases who had an excess were suffering from this complaint. It might, indeed, be said that a large proportion of persons affected with chronic gastric catarrh are gouty; but the same state of the saliva was present in those suffering from recent ulceration of the stomach, although they were restricted to a very meagre diet. We have before seen that the most common complaint amongst those whose saliva showed a deficiency was an atonic state of the digestive organs, and it now appears that inflammatory affections of the stomach are as constantly present in those in whom it is in excess; we have therefore a means of diagnosis between these states of the digestive canal which it is so often difficult to distinguish, but in which the successful treatment entirely depends upon the accuracy of the diagnosis.

As far as my observations have gone I should be inclined to state that all previously healthy individuals, when affected with an inflammatory disorder, present an excess of sulphocyanide during the acute attack, but that this is afterwards followed by a diminution. For example, of 9 cases of acute pleurisy 4 were examined within one week of the commencement of the illness, and 3 presented it in excess, and 1 in normal quantity; 3 were examined between the second and third week, and 2 had an excess and 1 less than normal; after the third week 2 were examined, and both were below normal.

Twenty-six cases of phthisis are recorded, of whom 25 per cent. had a deficiency of sulphocyanide when examined within six months of the beginning of the illness; 58 per cent. of those between the sixth and twelfth months; and 
no less than 87 per cent. of those above twelve months presented the same condition.

It was before shown that in rheumatic fever the amount of sulphocyanide bore some proportion to the height of the temperature and the rapidity of the pulse; but in phthisis the opposite is observed. Thus, of six cases below the normal amount the average temperature was $101^{\circ}$, whilst of seven whose saliva was normal and of six cases of excess, the average of each was 99.4 . It is evident, therefore, that the formation of sulphocyanide is not necessarily the result of a high temperature; and if we bear in mind the loss of appetite and the tendency to vomiting and diarrhoea that so constantly accompany the advanced stages of phthisis, we can have little difficulty in understanding why the sulphocyanide should be deficient at this period of the disease.

In diseases of the kidneys the excess is greatest at the outset of the illness; the average duration of those presenting +8 was $5 \frac{1}{2}$ weeks, those +4 was 5 months, and those +2 was 14 months.

Ten cases are registered as suffering from heart disease ; those under twelve months' duration had all an excess or a normal amount of the sulphocyanide; but after twelve months, where the digestive powers had become enfeebled, as is almost always the case in this malady, every case presented the sulphocyanide below the normal quantity.

The question arises whether there is any hypothesis by which we can explain the above observations. We have seen that in stout persons and in those gaining flesh the saliva usually contains an excess of sulphocyanide, and the most probable explanation seems to be that one of the functions of the liver in health is to break up any nutritive material that may be absorbed and is in excess of the requirements of the system. We know from the researches of Andral and others, that in the diseases in which an abnormal quantity of sulphocyanide has been discovered, viz. in rheumatic fever, erysipelas, and all acute inflammations, the albumen of the blood is so altered voL. LXV. 
that the quantity of the fibrin-forming material is greatly incrèased. May we not then suppose that the liver under these circumstances breaks up such of the albumen as has been rendered unfit for nutrition, and that the excess of sulphocyanide represents this increased destruction of this altered material? After a certain period, the duration of which varies in different diseases, the amount of albumen in the blood becomes insufficient for the requirements of nutrition, and then we find, as we have before stated, a diminution in the quantity of the sulphocyanide in the saliva.

I have stated the conclusions which seem to me to arise from the above-mentioned facts only as probable, because the chances of errors of observation are so much greater when only a "colour test" is employed than when the amount of a substance is quantatitively determined. The labour involved, however, in employing a more accurate method would have been so great, and the number of cases collected so few, that it seemed to me to be advisable to attempt to obtain in the first place only a general view of the subject, and to leave the settlement of any questions that might arise to future and more careful researches.

I have, I trust, been able to prove that the presence of the sulphocyanide in the saliva is not due to accidental circumstances, but is regulated, as is the case with the secretions of other glands, by the operation of general laws; and above all, that the whole subject, whether viewed in the light of its scientific interest or its practical importance, is worthy of more attention than has been hitherto bestowed upon it. 